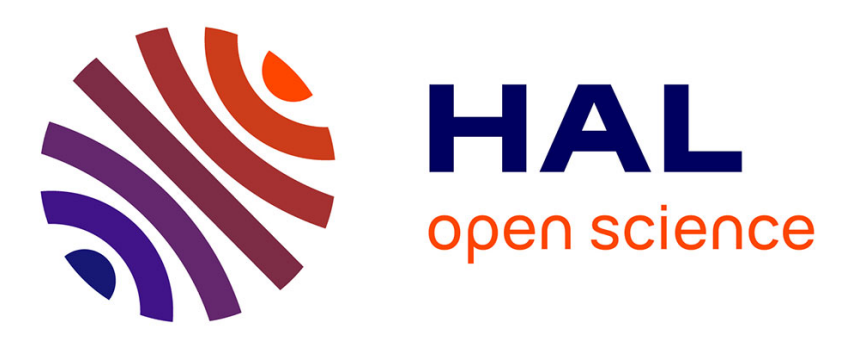

\title{
Engineering of geranylgeranyl pyrophosphate synthase levels and physiological conditions for enhanced carotenoid and astaxanthin synthesis in
}

Jürgen Breitenbach, Hans Visser, Jan C. Verdoes, Albert J. J. Ooyen, Gerhard Sandmann

\section{To cite this version:}

Jürgen Breitenbach, Hans Visser, Jan C. Verdoes, Albert J. J. Ooyen, Gerhard Sandmann. Engineering of geranylgeranyl pyrophosphate synthase levels and physiological conditions for enhanced carotenoid and astaxanthin synthesis in. Biotechnology Letters, 2010, 33 (4), pp.755-761. 10.1007/s10529-0100495-2 . hal-00653064

\section{HAL Id: hal-00653064 https://hal.science/hal-00653064}

Submitted on 17 Dec 2011

HAL is a multi-disciplinary open access archive for the deposit and dissemination of scientific research documents, whether they are published or not. The documents may come from teaching and research institutions in France or abroad, or from public or private research centers.
L'archive ouverte pluridisciplinaire HAL, est destinée au dépôt et à la diffusion de documents scientifiques de niveau recherche, publiés ou non, émanant des établissements d'enseignement et de recherche français ou étrangers, des laboratoires publics ou privés. 
Section: Microbial and Enzyme Technology

Engineering of geranylgeranyl pyrophosphate synthase levels and physiological conditions for enhanced carotenoid and astaxanthin synthesis in Xanthophyllomyces dendrorhous.

Jürgen Breitenbach, Hans Visser, Jan C. Verdoes, Albert J.J. van Ooyen, Gerhard Sandmann

J. Breitenbach, G. Sandmann*

J.W. Goethe Universität, Molecular Biosciences 213, P.O. Box 111932, 60054 Frankfurt, Germany;

E-mail:sandmann@em.uni-frankfurt.de

Tel.:+49-69-79824746

Fax:+49-69-79824822

J.C. Verdoes**

Division of Industrial Microbiology

Wageningen University, P.O. Box 8129, 6700 EV Wageningen, The Netherlands

H. Visser, A.J.J. van Ooyen

Section of Fungal Genomics, Wageningen University, Dreijenlaan 2, 6703 HA

Wageningen, The Netherlands

Present address:

H. Visser

Dyadic Netherlands, Nieuwe Kanaal 7-s, 6709 PA Wageningen, The Netherlands

*corresponding author

$* *$ deceased 
Abstract The basidiomycetous yeast, Xanthophyllomyces dendrorhous, is one of the very few organisms which can be used for biological production of the carotenoid astaxanthin. crtE cDNA has been cloned from this fungus for engineering of the terpenoid pathway. The function of its gene product as a geranylgeranyl pyrophosphate synthase was established. .X. dendrorhous was transformed with the $c r t E$ cDNA to divert metabolite flow from the sterol pathway towards carotenoid biosynthesis. Transformants were obtained with increased levels of geranylgeranyl pyrophosphate synthase leading to higher carotenoid levels including astaxanthin. Physiological conditions for maximum carotenoid synthesis for wild type and the CrtE transformant were dim light and extra air supply of the shaking culture. These conditions and the transformation with $\operatorname{crt} E$ had additive effects and resulted in an 8-fold higher astaxanthin formation as compared to the initial wild type culture without illumination and extra air supply yielding $451 \mu \mathrm{g} / \mathrm{g}$ dry wt within four days of growth.

Key words carotenoid biosynthesis; 3-hydroxy-4-keto-torulene; geranylgeranyl pyrophosphate synthase; light enhancement.

\section{Introduction}

Astaxanthin (3,3'-hydroxy-4,4'-diketo- $\beta$-carotene) is an industrially important carotenoid. It is used as feed additive in salmon and trout farming (Bjerkeng 2008) and has beneficial effects on human health (Hussein et al. 2006). The basidiomycetous yeast, Xanthophyllomyces dendrorhous (the sexual state of Phaffia rhodozyma), is the only fungus which synthesizes substantial amounts of astaxanthin (Andrewes and Starr 1976). Its carotenoid pathway is well established. The major end product is astaxanthin. However, a second product is 3-hydroxy-4keto-torulene (Vasquez and Santos 1998). This keto carotenoid is formed as a by-product (see Fig. 1 for details). Only three genes cover the whole specific carotenoid pathway from phytoene synthesis to the end products. These are $\operatorname{crt} Y B$, a fusion gene encoding a phytoene synthase and a lycopene cyclase (Verdoes et al. 1999a), crtI encoding a phytoene desaturase which forms lycopene and 3,4-didehydrolycopene (Verdoes et al. 1999b) and asy encoding an astaxanthin synthase which converts $\beta$-carotene via echineneone (4-keto- $\beta$-carotene), 3 hydroxy4-keto- $\beta$-carotene and phoenicoxanthin (3-hydroxy-4,4'-diketo- $\beta$-carotene to 
astaxanthin (Ojima et a. 2006). The function of the latter enzyme is dependent on a specific astaxanthin synthase reductase (Alcaíno et al. 2008).

An alternative or complementary approach to random mutagenesis for high yield strains (An et al. 1989) is genetic engineering of the astaxanthin synthesis pathway. In general, genetic modification may increase the flow of precursors into a specific pathway, mediate an efficient conversion of intermediates into the end product and divert a pathway at a branch point much stronger into a desired direction.

First attempts have been made for successful genetic engineering of the carotenoid pathway of $X$. dendrorhous. Introduction of extra copies of the $\operatorname{crtYB}$ fusion gene resulted in transformants with higher total carotenoid content (Verdoes et al. 2003a). This is due to the additional phytoene synthase activity. A second effect observed in the crtYB transformants was a change of the carotenoid composition with a strong decrease of 3-hydroxy-4ketotorulene, the alternative product of $X$. dendrorhous carotenoid biosynthesis. This can be attributed to the lycopene cyclase part of $\operatorname{crt} Y B$ gene product.

Feeding of the terpenoid precursor mevalonate to $X$. dendrorhous resulted in a fourfold increase of astaxantin content (Calo et al. 1995). Consequently, we regarded synthesis of GGPP as a substrate for phytoene as a limiting step for carotenoid synthesis as found before for Saccharomyces cerevisiae (Verwaal et al. 2007). Therefore, we engineered $X$. dendrorhous by over-expressing its own GGPP synthase gene. The resulting increase of astaxanthin formation was analyzed under different physiological conditions i.e. by variation of $\mathrm{O}_{2}$ supply and illumination.

\section{Materials and methods}

Strains, growth and physiological conditions

Xanthophyllomyces dendrorhous strain CBS6938 (=ATCC96594) was grown in $150 \mathrm{ml}$ of a medium containing Bacto-peptone ( $5 \mathrm{~g} / \mathrm{l})$, yeast extract $(3 \mathrm{~g} / \mathrm{l})$, malt extract $(3 \mathrm{~g} / \mathrm{l})$, and glucose $(10 \mathrm{~g} / \mathrm{l})$ in 11 conical flasks at $20^{\circ} \mathrm{C}$ on a rotary shaker for 4 days in darkness. Selection of transformants for G418 resistance was with geneticin (G-418 sulfate, $40 \mu \mathrm{g} / \mathrm{ml}$ ) containing agar plates. Light cultures were illuminated with low white light of $1 \mu \mathrm{mol}$ photons $\mathrm{m}^{-2} \mathrm{~s}^{-1}$. High $\mathrm{O}_{2}$ cultures were supplemented with extra air at $50 \mathrm{ml} / \mathrm{min}$. 
Transformation of $X$. dendrorhous was carried out by electroporation according to Visser et al. (2005).

Escherichia coli strains DH5 $\alpha$, XL1-Blue MFR and JM101 used for genetic manipulations or functional complementation, respectively, were grown on LB medium with ampicillin (100 $\mu \mathrm{g} / \mathrm{ml})$ and chloramphenicol (34 mg/l).

Cloning of the GGPP synthase cDNA and construction of plasmids for E. coli and $X$. dendrorhous

A cDNA library from $X$. dendrorhous (Ojima et al. 2006) in E. coli XL1-Blue MFR was screened for a GGPP synthase cDNA by functional complementation and color selection. For this purpose, the library was co-transformed with plasmid pACCAR25 $\Delta$ crtE (Sandmann et al. 1993), which contains all the genes for the synthesis of zeaxanthin glucosides except the gene for GGPP synthase $\operatorname{crtE}$. Functional complementation with a GGPP synthase cDNA should enable the synthesis of colored carotenoids. An orange pigmented colony was found among 8000 white ones. This $X$. dendrorhous cDNA was used as template for further plasmids. Plasmid pUC8-2crtE was designed for $c r t E$ expression in E. coli. It was constructed by PCR amplification with primers EcocrtEforw 5'-GAATTCATGGATTACGCGAACATCCTC-3' and crtErew 5'-GTCGACTCACAGAGGGATATCGGCTAG-3', and sub-cloned into Toverhang vector pMON38201 (Borovkov and Rivkin 1997). The crtE insert was subsequently excised with EcoRI and PstI and finally ligated into pUC8-2 (Hanna et al. 1984). The plasmid with the crtE cDNA for transformation of $X$. dendrorhous was obtained by amplification with the primers PcrtEATG 5'-ATATGAATTCATGGATTACGCGAACATCCTC-3' and PcrtETGA 5'-TATAGTCGACTCACAGAGGGATATCGGCTAG-3' which generated EcoRI and SalI cloning sites. The obtained PCR product of expected size $(1.1 \mathrm{~kb})$ was ligated into the EcoRI and XhoI sites of vector pPRcDNA1 (Ojima et al. 2006) yielding pPRcDNA1 crtE.

Carotenoid extraction and analysis

Cells of $X$. dendrorhous were harvested and freeze-dried. Carotenoids were subsequently extracted from these cells with dimethylsulfoxide by heating for $15 \mathrm{~min}$ at $60^{\circ} \mathrm{C}$. After centrifugation, the extract was transferred into a separator funnel, diethyl ether was added, and the mixture was kept on ice for $3 \mathrm{~min}$ before water was added for phase separation. The upper phase was collected to which step by step acetone, $10 \%(\mathrm{v} / \mathrm{v})$ diethyl ether in petroleum 
ether (bp $60-80^{\circ} \mathrm{C}$ ) and water were added. Again, the upper phase was collected, washed with water, dried in a stream of $\mathrm{N}_{2}$, and re-suspended in acetone. Details of the extraction procedure are given elsewhere (Visser et al. 2005).

Freeze-dried E. coli cells were extracted with methanol by heating for $15 \mathrm{~min}$ at $60^{\circ} \mathrm{C}$. The extract was partitioned against 50\% (v/v) ether in petrol and the upper phase collected and dried. High-performance liquid chromatography (HPLC) separation of carotenoids was performed on a $25 \mathrm{~cm}$ HyPurity $\mathrm{C} 18,5 \mu$ column with acetonitrile /methanol/2-propanol (85:10:5, by vol) as the mobile phase. Spectra were recorded on-line with a photodiode array detector. Carotenoid identification and quantitation was carried out with authentic standards.

\section{Results and discussion}

Function of $c r t E$ and the $X$. dendrorhous crtE transformation vector

The cloned GGPP synthase cDNA crtE from X. dendrorhous encoded a protein of 375 amino acids. The sequence of the cDNA and genomic DNA is presented elsewhere (van Ooijen et al. 2007). The function of the cDNA was determined by pathway complementation in E. coli using plasmid pACCAR25 $\Delta \mathrm{crtE}$, which contained all genes for the synthesis of zeaxanthin diglucoside except the GGPP synthase gene (Sandmann et al. 1993). Upon separation by HPLC, only minor amounts of zeaxanthin diglucoside and zeaxanthin of together $4 \mu \mathrm{g} / \mathrm{g} \mathrm{dw}$ were detectable due to the very low GGPP pool in E. coli. When E. coli was co-transformed with pUC8-2crtE (for vector map see Fig. 2 top), which was designed for $\operatorname{crt} E$ expression, the concentration of the same carotenoids including small amounts of zeaxanthin monoglucoside increased more than 30 -fold to $128 \mu \mathrm{g} / \mathrm{g} \mathrm{dw}$. This result indicated that the $\mathrm{CrtE}$ from $X$. dendrorhous indeed is the GGPP synthase as shown in Fig. 1.

After assignment of the $c r t E$ cDNA as a GGPP synthase, it was used to construct a transformation plasmid for crtE genome integration and over-expression in X. dendrorhous. The resulting plasmid is shown in Fig. 2 bottom. Both the crtE cDNA and the nptII gene conferring resistance of transformed $X$. dendrorhous to geneticin (G418) were flanked by the promoter and terminator of the $X$. dendrorhous glyceraldehyde-3-phosphate dehydrogenase gene. The $\operatorname{crtE}$ transformation vector contained a ribosomal DNA sequence region for multiple copy integration into the $X$. dendrorhous genome. Integration of the transformation 
vector was achieved after linearization by the single-cutting restriction enzyme SfiI prior to transformation.

Carotenoid formation in $X$. dendrorhous CBS6938[crtE] transformants

Since after transformation of $X$. dendrorhous, variations in the resulting phenotype can be found depending on the number of integrated copies (Verdoes et al. 2003), different CBS6938[crtE] transformants were selected according to the intensity of their pigmentation. The carotenoids of the three most pigmented transformants are shown in Table 1. They synthesize between 1.3 - to 1.5 -fold more total carotenoids than the control reaching up to 288 $\mu \mathrm{g} / \mathrm{g}$ dry weight. Transcript determinations of $\mathrm{crt} E$ in transformant no. 1 revealed a 10 times higher level than in the wild type strain (data not shown). In spite of the resulting enhanced overall carotenoid synthesis, formation of the pathway end product astaxanthin did not increase accordingly. Instead, also keto carotenoid intermediates accumulate. This result indicates that the transformants have a certain limitation in the biosynthesis pathway to astaxanthin for the last steps catalyzed by Asy (Fig. 1). A similar observation was also reported for $X$. dendrorhous transformants that over-expressed the crtYB gene (Verdoes et al. 2003).

The major terpenoid in fungi is ergosterol. Its synthesis branches off the route to carotenoids at the level of farnesyl pyrophosphate, the substrate for GGPP synthase. The concentrations of ergosterol and carotenoids including colorless phytoene were determined in wild type CBS6938 and the transformant CBS6938[crtE] with over-expressed GGPP synthase (Table 2). In the wild type strain grown without extra air supply, the carotenoid content was substantially lower than ergosterol regardless whether permanently illuminated or not. Enhanced air supply increased colored carotenoids at the expense of ergosterol. Additionally, the phytoene content decreased. These results suggest a better utilization of FPP towards carotenogenesis and a more efficient conversion within the carotenoid pathway towards the end products. This up-regulation of astaxanthin synthesis with higher $\mathrm{O}_{2}$ supply may be due to generated singlet oxygen and oxygen radicals (Schroeder and Johnson 1995). Another environmental factor which can stimulate carotenogenesis in $X$. dendrorhous is light (de la Fuente et al. 2010). With our wild type strain, the highest astaxanthin and 3-hydroxy-4ketotorulene concentrations were obtained in the light with extra air supply, which with 452 $\mu \mathrm{g} / \mathrm{d}$ dry weight were 5-fold higher than in the dark, culture without extra air. The same tendency was observed for the $c r t E$ transformant. Here, we obtained an additive effect 
between GGPP synthase over-expression, illumination and $\mathrm{O}_{2}$ supply, which all contributed to increased synthesis of total carotenoids including astaxanthin. In the case of astaxanthin, the yield was 8-fold higher than in the initial wild type culture without illumination and extra air supply reaching a maximum of $451 \mu \mathrm{g} / \mathrm{g}$ dry wt.

\section{Conclusion}

Over-expression of $c r t E$ is a useful target to divert and enhance metabolite flow into carotenogenesis. In addition, physiological conditions like dim light illumination of and enhanced air supply to the shaking culture all independently contributed to enhanced astaxanthin synthesis in an additive way. Although astaxanthin yield could be strongly increased, its amount accounts only for $43 \%$ of total carotenoids even under optimum conditions. Most of the residual carotenoids are keto derivatives which are not completely metabolized into astaxanthin. This finding suggests that asy over-expression should be the next step to increase astaxanthin yield. Previous transformation with additional copies of the crtYB gene was already successful in increasing carotenoid formation (Verdoes et al. 2003). Therefore, maximum astaxanthin biosynthesis can finally be achieved both by generating a fully genetically engineered $X$. dendrorhous with additionally expressed $\operatorname{crt} E, \operatorname{crt} Y B$ and $a s y$ genes which are likely to alleviate limitations of the carotenoid pathway in combination with advantageous cultivation conditions. This overall genetic approach will represent a decisive step towards the development of $X$. dendrorhous as a cell factory for astaxanthin production which may economically compete with chemical synthesis.

\section{References}

Alcaino J, Barahona S, Carmona M, Lozano C, Marcoleta A, Niklitschek M, Sepulveda D, Baeza M, Cifuentes V (2008) Cloning of the cytochrome P450 reductase (crtR) gene and its involvement in the astaxanthin biosynthesis of Xanthophyllomyces dendrorhous. BMC Microbiology 8:169

An GH, Schuman DB, Johnson EA (1989) Isolation of Phaffia rhodozyma mutants with increased astaxanthin content. Appl Environ Microbiol 55:116-124 
Andrewes AG, Starr MP (1976) (3R,3'R)-Astaxanthin from the yeast Phaffia rhodozyma.

Phytochemistry 15:1009-1011

Bjerkeng B (2008) Carotenoids in aquaculture: Fish and Crustaceans. In: Britton, G., LiaaenJensen, S., Pfander H. (eds) Carotenoids Vol. 4, pp.237-254, Birkhäuser Verlag, Basel, $\mathrm{CH}$

Borovkov AY, Rivkin MI (1997) XcmI-Containing vector for direct cloning of PCR products, BioTechn 22:812-814

Calo P, de Miguel T, Velazquez JB, Villa TG (1995) Mevalonic acid increases transastaxanthin and carotenoid biosynthesis in Phaffia rhodozyma. Biotechnol Lett $17: 575-578$

de la Fuente JL, Rodríguez-Sáiz M, Schleissner C, Díez B, Peiro E, Barredo JL (2010) Hightiter production of astaxanthin by the semi-industrial fermentation of Xanthophyllomyces dendrorhous. J Biotechnol 148:144-146

Hanna Z, Fregau C, Prefontaine G, Brousseau R (1984) Construction of a family of universal expression plasmid vectors. Gene 30:247-250

Hussein G, Sankawa U, Goto H, Matsumoto K, Watanabe H (2006) Astaxanthin, a carotenoid with potential in human health and nutrition. J Nat Prod 69:443-449

Ojima K, Breitenbach J, Visser H, Setoguchi Y, Tabata K, Hoshino T, van den Berg J, Sandmann G (2006) Cloning of the astaxanthin synthase gene from Xanthophyllomyces dendrorhous (Phaffia rhodozyma) and its assignment as a betacarotene 3-hydroxylase / 4-ketolase. Mol Gen Genom 275:148-158

van Ooijen AJJ, Verdoes JC, Wery J (2007) Recombinant material for carotenoid production. US patent no. 7,205,123 B2

Sandmann G, Misawa N, Wiedemann W, Vittorioso P, Carattoli A, Morelli G, Macino G (1993) Functional identification of al-3 from Neurospora crassa as the gene for geranylgeranyl pyrophosphate synthase by complementation with crt genes, in vitro characterization of the gene product, and mutant analysis. J Photochem Photobiol B: Biol 18:245-251

Schroeder WA, Johnson EA (1995) Singlet oxygen and peroxyl radicals regulate carotenoid biosynthesis in Phaffia rhodozyma. J Biol Chem 270:18374-18379

Vazquez M, Santos V (1998) 3-Hydroxy-3', 4'-didehydro-beta- $\psi$-caroten-4-one (HDCO) from Xanthophyllomyces dendrorhous (Phaffia rhodozyma) cultivated on xylose media. Biotechnol Lett 20:181-182 
Verdoes JC, Krubasik KP, Sandmann G, van Ooyen AJJ (1999a) Isolation and functional characterisation of a novel type of carotenoid biosynthetic gene from Xanthophyllomyces dendrorhous. Mol Gen Genet 262:453-461

Verdoes JC, Misawa N, van Ooyen AJJ (1999b) Cloning and characterization of the astaxanthin biosynthetic gene encoding phytoene desaturase of Xanthophyllomyces dendrorhous. Biotechnol Bioengin 63:750-755

Verdoes JC, Sandmann G, Visser H, Diaz M, van Mossel M, van Ooyen AJJ (2003) Metabolic engineering of the carotenoid biosynthetic pathway in the yeast Xanthophyllomyces dendrorhous (Phaffia rhodozyma). Appl Environm Microbiol 69:3728-3738

Verwaal R, Wang J, Meijnen JP, Visser H, Sandmann G, van den Berg JA, van Ooyen AJJ (2007) High level production of beta-carotene in Saccharomyces cerevisiae by successive transformation with carotenogenic genes from Xanthophyllomyces dendrorhous. Appl Environ Microbiol 73:4342-4350

Visser H, Sandmann G, Verdoes JC (2005) Xantophylls in fungi: metabolic engineering of the astaxanthin biosynthetic pathway in Xantophyllomyces dendrorhous. In: Barredo J (ed) Methods in biotechnology pp 257-272. Microbial Processes and Products. Totowa, NJ, USA 
Table 1 Total colored carotenoids and \% distribution of individual carotenoids in Xanthophyllomyces dendrorhous wild type CBS6938 (control) and individual crtE transformants CBS6938[crtE] (numbers 1, 2, 3).

Carotenoids $(\mu \mathrm{g} / \mathrm{g} \mathrm{dw})$

\begin{tabular}{|c|c|c|c|}
\hline Control & 1 & 2 & 3 \\
\hline $198 \pm 38$ & $288 \pm 56$ & $263 \pm 50$ & $259 \pm 57$ \\
\hline
\end{tabular}

\% Distribution

\begin{tabular}{|c|c|c|c|c|}
\hline Astaxanthin & 37.5 & 29.1 & 32.4 & 28.6 \\
\hline Hydroxycanthaxanthin & 13.8 & 12.8 & 18.0 & 19.4 \\
\hline Hydroxyketotorulene & 15.7 & 27.7 & 21.2 & 19.5 \\
\hline Hydroxyechinenone & 11.3 & 12.1 & 6.1 & 13.4 \\
\hline Echinenone & 8.7 & 5.3 & 7.4 & 8.3 \\
\hline Lycopene & 7.0 & 0 & 2.2 & 0 \\
\hline Torulene & 0.5 & 1.6 & 3.8 & 4.0 \\
\hline B-Carotene & 6.5 & 11.4 & 8.8 & 6.8 \\
\hline
\end{tabular}

Growth was for 4 days without extra air supply, in the dark at $20^{\circ} \mathrm{C}$ as shaking cultures. Means \pm standard deviation were from 3 to 5 determinations. 
Table 2 Ergosterol and carotenoid formation ( $\mu \mathrm{g} / \mathrm{g} \mathrm{dw})$ in wild type Xanthophyllomyces dendrorhous CBS6938 and a transformant with integrated crtE gene CBS6938[crtE]\#1

Conditions Ergosterol Phytoene TCC 3HO4KT Astaxanthin

CBS6938

$\begin{array}{lcllcc}\text { dark, LO } & 1060 \pm 257 & 84 \pm 13 & 167 \pm 11 & 16 \pm 7 & 57 \pm 8 \\ \text { dark, HO } & 864 \pm 342 & 5 \pm 3 & 602 \pm 28 & 53 \pm 9 & 147 \pm 11 \\ \text { light, LO } & 1014 \pm 208 & 57 \pm 5 & 223 \pm 17 & 20 \pm 4 & 56 \pm 8 \\ \text { light, HO } & 975 \pm 333 & 10 \pm 7 & 694 \pm 49 & 87 \pm 12 & 304 \pm 29\end{array}$

CBS6938[crtE]

$\begin{array}{llllll}\text { dark, LO } & 1042 \pm 554 & 104 \pm 20 & 244 \pm 18 & .6 \pm 7 & 77 \pm 19.6 \\ \text { dark, HO } & 881 \pm 206 & 50 \pm \pm 6 & 963 \pm 54 & 106 \pm 14 & 250 \pm 32 \\ \text { light, LO } & 1029 \pm 164 & 98 \pm 11 & 369 \pm 43 & 20 \pm 8 & 111 \pm 8 \\ \text { light, HO } & 916 \pm 104 & 40 \pm 15 & 1007 \pm 65 & 126 \pm 13 & 452 \pm 44\end{array}$

LO, low $\mathrm{O}_{2}=$ without extra air supply; $\mathrm{HO}$, high $\mathrm{O}_{2}=$ with extra air supply; TCC, total colored carotenoids; $3 \mathrm{HO} 4 \mathrm{KT}$, 3-Hydroxy-4-keto-torulene; means \pm standard deviation from 3 to 5 determinations; 


\section{figure legends}

Fig. 1 Carotenogenic pathway of Xanthophyllomyces dendrorhous and sites (bold arrows) for metabolic engineering of astaxanthin synthesis. The gene products catalyzing the individual reactions are indicated: $\mathrm{CrtE}$, geranylgeranyl pyrophosphate synthase; $\mathrm{CrtYB}$, phytoene synthase / lycopene cyclase; CrtI, phytoene desaturase; Asy, astaxanthin synthase. All final reactions in the oval are catalyzed by astaxanthin synthase. Abbreviations: FPP, farnesyl pyrophosphate; GGPP geranylgeranyl pyrophosphate.

Fig. 2 Transformation vectors pUC8-2crtE for CrtE expression in Escherichia coli (top) and pPRcDNA1 crtE for over-expression of geranylgeranyl pyrophosphate synthase in Xanthophyllomyces dendrorhous (bottom). 
Precursors

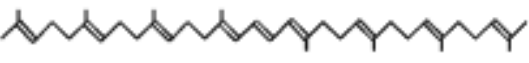

Phytoene $\downarrow$
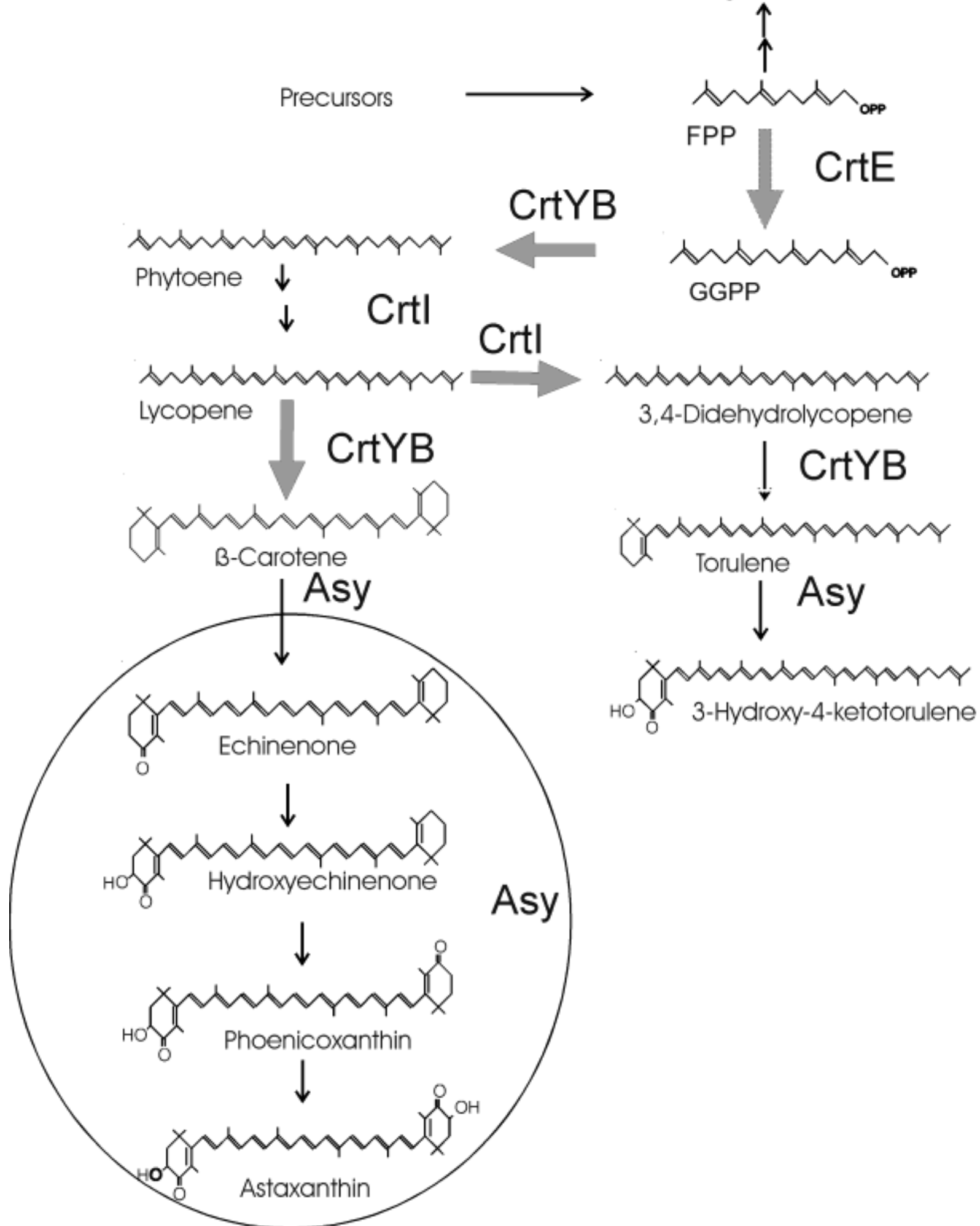

Ergosterol 

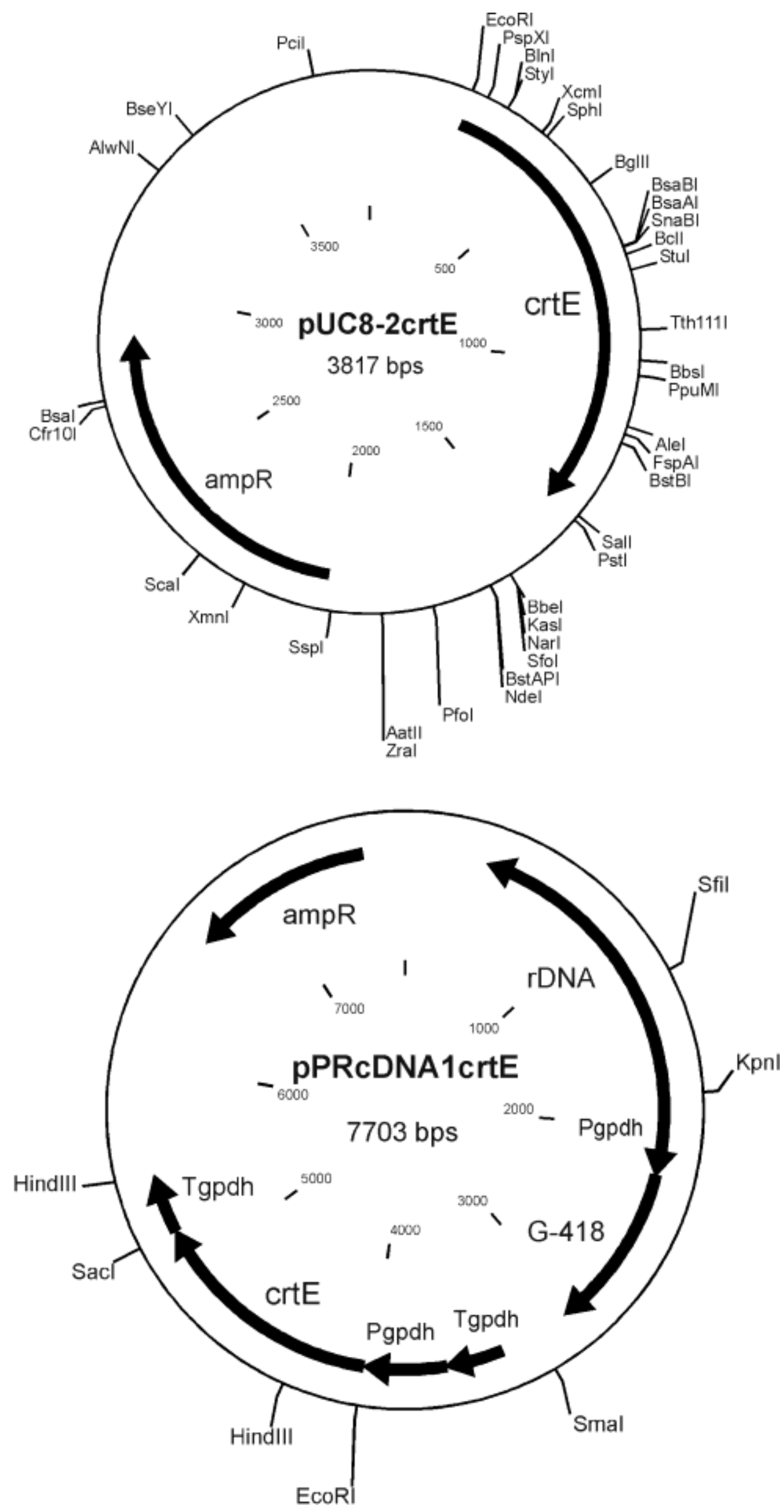